ARGONNE NATIONAL LABORATORY

9700 South Gass Avenue

Argonne, Illinors 60439

\title{
SOME COMMENTS ON
}

THE POWER-BALANCE PARAMETERS $Q$ AND $\&$

AS MEASURES OF PERFORMANCE

FOR FUSION POWER REACTORS

by

P. J. Persiani

Applied Physics Division

W. C. Lipınski

Reactor Analysis and Safety Division

A. J. Hatch

Physies Division

י.

June 1972

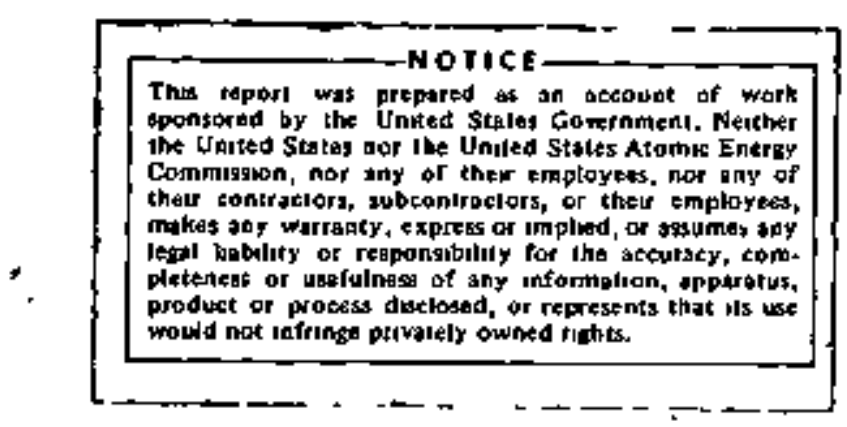




\section{DISCLAIMER}

This report was prepared as an account of work sponsored by an agency of the United States Government. Neither the United States Government nor any agency Thereot, nor any of their employees, makes any warranty, express or implied, or assumes any legal liability or responsibility for the accuracy, completeness, or usefulness of any information, apparatus, product, or process disclosed, or represents that its use would not infringe privately owned rights. Reference herein to any specific commercial product, process, or service by trade name, trademark, manufacturer, or otherwise does not necessarily constitute or imply its endorsement, recommendation, or favoring by the United States Government or any agency thereof. The views and opinions of authors expressed herein do not necessarlly state or reflect those of the United States Government or any agency thereof. 


\section{DISCLAIMER}

Portions of this document may be illegible in electronic image products. Images are produced from the best available original document. 
TABLE OF CONTENTS

Page

ABSTRACT ..................... 5

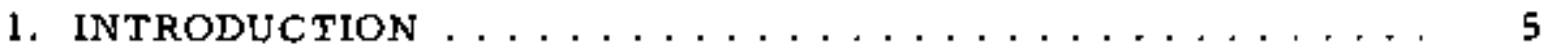

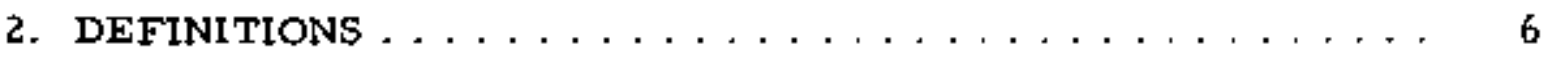

3. LIMIT TESTS . . . . . . . . . . . . . . . . . 9

4. COMPARISON WITH OTHER $Q$ DEFINITIONS $\ldots \ldots \ldots \ldots 10$

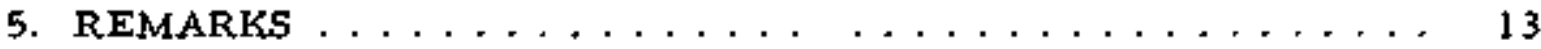

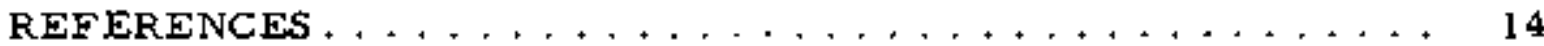




\section{LIST OF FIGURES}

No.

Title

Page

1, Basic Power flow Diagram ................... 6

2. Typical Power-flow Diagram ................. 8

\section{TABLE}

No.

Title

I. Values of $Q$ and $e$ for Mirror Reactors............ 


\title{
SOME COMMENTS ON \\ THE POWER-BALANCE PARAMETERS Q AND \& \\ AS MEASURES OF PERFORMANCE \\ FOR FUSION POWER REACTORS
}

by

\author{
P. J. Persiani, W. C. Lipinski, \\ and A. J. Hatch
}

\begin{abstract}
Power-producing fusion-reactor systems may require a complex of plasma-producing subsyetema (containment, heating, and injection) and of energy-conversion subsystems (thermal and direct). To establish a basis for a comparison of these reactor power syztems, generalıed definitions based on power balance are proposed for the two important power-balance parameters: (1) $Q$ (ratio of output to input power), and (2) e (fractional circulating power). The valuea of $Q$ and $c$ determined from the proposed generalized definitions are compared with values in the literature based on different definitions.
\end{abstract}

\section{INTRODUCTION}

A survey of analytical studies of power-producing fusion reactors ${ }^{\prime}$ indicates that some reactor systems may require a complex of plasmaproducing subsystems (containment, heating, and injection) and of energyconversion subsystems (thermal and direct). The power-balance parameters used to characterize the behavior of some of these reactor systems are $Q$ and $\varepsilon$. However, there are significant basic differences in the tefinitions as given by various authors. To establish a common basis for a comparison of theae complex power systems, it has been found useful to define $Q$ and $\varepsilon$ based on a power balance of the total reactor system. The definitions are developed in Sec. $Z_{i}$ limit tests and other considerations that demonstrate the generalized nature of these definitions are applied in Sec. 3; the $Q$ values computed from the various definitions are compared in Sec. 4; and concluding remarks are contained in Sec. 5. 


\section{DEFINITIONS}

The relationship between the subsystems of a power reactor system is shown schematically in the basic power-flow diagram of Fig. 1. The power-balance equation for a reactor system may be written as the product form of the ratios of power output to power input for each of the subsystems

$$
\frac{P_{i}}{P_{c}} \times \frac{P_{o}}{P_{i}} \times \frac{P_{g}}{P_{o}} \times \frac{P_{c}}{P_{g}}=1,
$$

where

$$
\begin{aligned}
& P_{i} \equiv \text { power input to the power-source subsystem, } \\
& P_{0} \equiv \text { power output from the power-source subsystem, } \\
& P_{g} \equiv \begin{array}{l}
\text { gross electrical power output from the power-conversion } \\
\text { subsystem. }
\end{array}
\end{aligned}
$$

and

$$
\begin{aligned}
& P_{c} \bar{F} \text { circulating electrical power to the plasma-preparation } \\
& \text { subsystem. }
\end{aligned}
$$

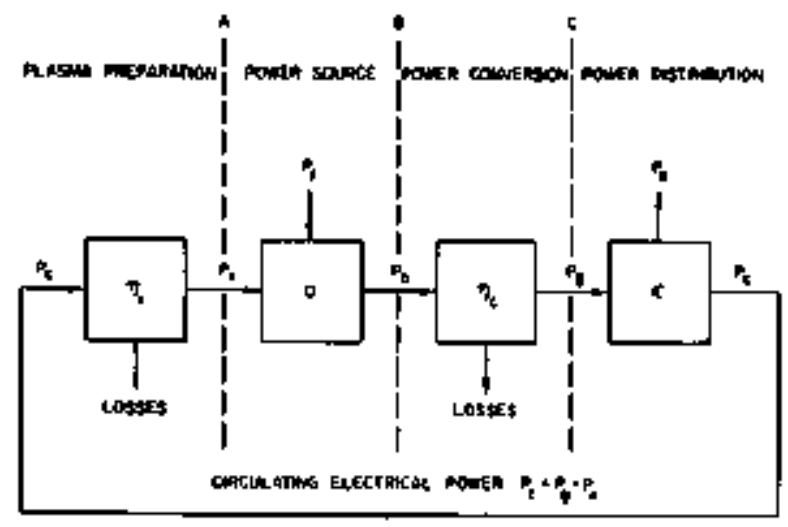

Fir. 1

Basic Power-flow Diagram. ANL Neg. No. 116-1080.

In Fig. 1, the quantity $P_{C}-P_{i}$ is the power loss of the plasmapreparation subsystem having an efficiency $\eta_{i}, P_{o}-P_{g}$ is the power loss of the conversion subsystem having an efficiency $\eta_{c}$, and $P_{g}-P_{c}$ is the net electrical power output $P_{\text {e }}$ available for external distribution. With the potwer multiplication factor $Q$ of the power source subsystem defined as

$$
Q=\frac{P_{o}}{P_{i}}
$$

and the fractional circulating power $\&$ defined as

$$
\epsilon=\frac{\mathrm{P}_{\mathrm{c}}}{\mathrm{P}_{\mathrm{g}}}
$$


the power-balance relation given by Eq. 1 may be written as

$$
Q=\frac{1}{e \eta_{i} \eta_{c}}
$$

where $\eta_{i}=P_{i} / P_{c}$ and $\eta_{c}=P_{g} / P_{a}$. For an overall system efficiency $\eta_{1}$ defined as the product of $\eta_{i}$ and $\eta_{c}$, Eq. 4 may be further reduced to

$$
Q \frac{j}{1} \frac{1}{\varepsilon \eta}
$$

The $Q$ factor as defined in Eq. 2 is the ratio of the total reactor power output $P_{0}$ (across interface $B$ ) to the total power input $P_{i}$ (across interíce A), where

$$
P_{Q} i=P_{i}+P_{i} .
$$

and where $P_{f}$ is the fusion power generated in the power-source subsystem. Note that the conservation of energy requires that the output power must include the input power. The parameter $Q$ may also be written as

$$
\mathrm{Q}=1+\frac{\vec{P}_{\mathbf{f}}}{\vec{P}_{\mathrm{i}}}
$$

As delined here, $Q$ corresponds to the quantity $R+1$ used by Lawson ${ }^{2}$ in developing the break-even criterion for a thermonuclear reactor. The parameter a may also be written in the alternative useful forms

$$
\varepsilon=1-\frac{P_{\mathrm{e}}}{\mathrm{P}_{\mathrm{g}}}
$$

and

$$
\varepsilon=\frac{1}{1+P_{e} / P_{c}}
$$

For the power-source subsystem alone, the lower-limiting condition for $P_{O}=P_{i}$ corresponds to $Q=1$ in Eq. 2. If $P_{0}$ exceeds $P_{i}$ (i.e., the subsystem is a power producer), then $Q>1$ and the upper-limiting value $Q=\infty$ occurs if $P_{i}=0$ (with $P_{0}$ finite). Therefore, the $Q$ factor assumes a range of values

$$
1 \leq Q \leq \infty .
$$

As shown in Fig. 1, $P_{g}$ is the total power from the power-conversion subsystem (across interface $C$ ), and $P_{C}$ is the power flow into the plasmapreparation subsystem. For a power-producing system, the range of $P_{C}$ is $0 \leq P_{C} \leq P_{g}$; therefore from Eq. 3 the range of $q$ becomes

$$
0 \leq \varepsilon \leq 1 \text {. }
$$


The lower value corresponds to the condition that all the gross electrical power output is available for external distribution (i.e., the circulating power is zero). The upper value corresponds to a system in which the total gross electrical power output must be circulated to maintain the system (i.e., the net electrical power output is zero). If the system is not self-sustaining, the power deficiency must be made up by an external electrical power source in order to maintain operation. Under these circumstances, $\epsilon$ is greater than unity, corresponding to a negative $P_{e}$. which implies an input power from an external system. Present experimental plasma systems operate in this range.

The significance of Eq. 4 for $Q$ is that it incorporates $a$ and the efficiencies of the various subsystems for the entire reactor system. The relation states that for a $Q$ value determined by the characteristics of the power-source subsystem, the fractional citculating power can be reduced only by increasing the efficiencies of the plasma-preparation and/or powerconversion subsystems.

A more detailed power-flow diagram of a typical fusion-powerreactor system is shown in Fig. 2. The complete reactor system is divided into four major subsystems: plasma preparation, power source. power conversion, and power distribution. The plasma-preparation subsystem is further subdivided into two minor subsystems, and the energyconversion subsystem is divided into three minor subsystems.

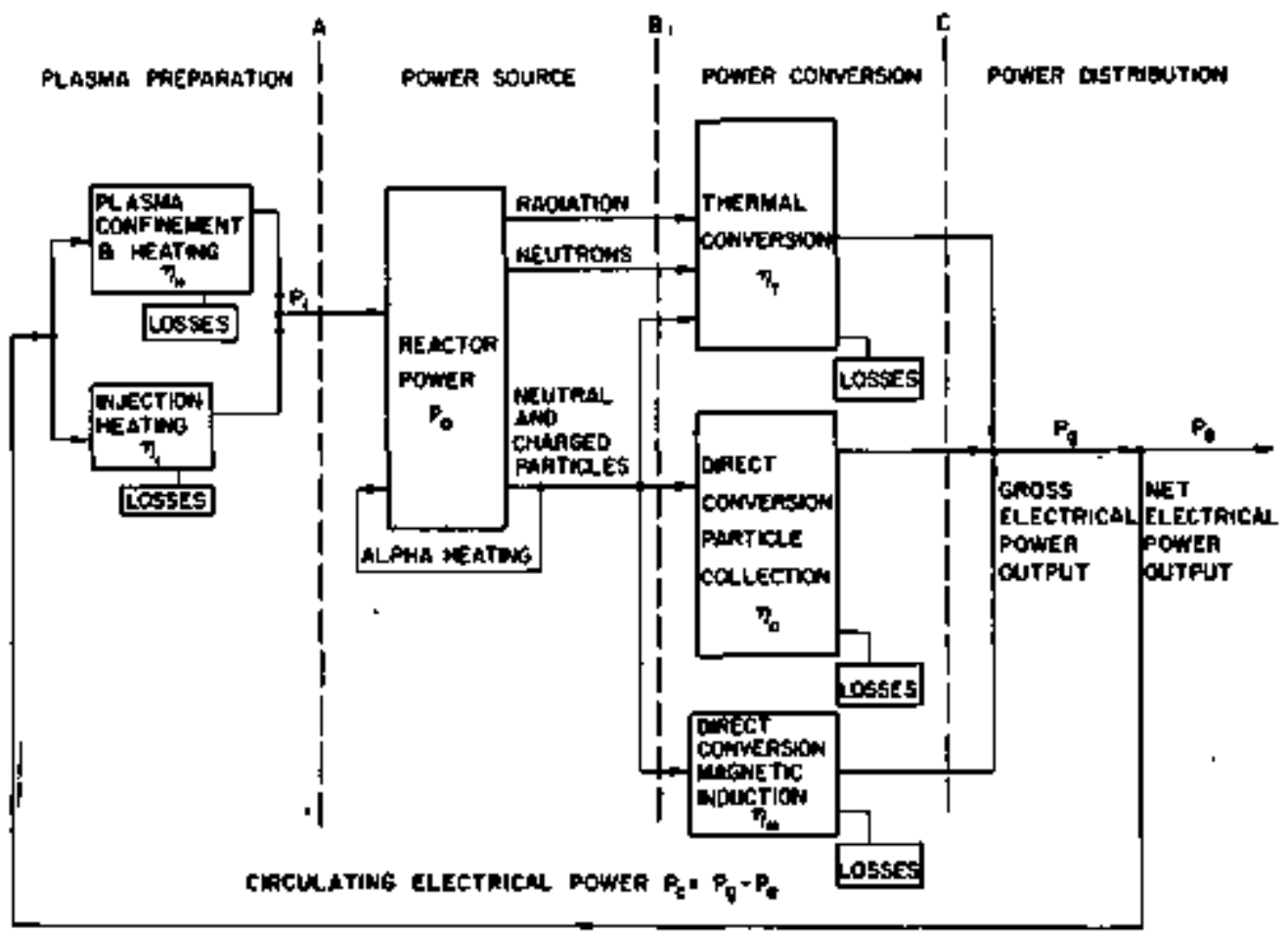

Fig. 2. Typical Power-flow Diagram. ANL Neg. No, 116-743 Rev. 2.

Wor shown explicity in the diagram but implicit in the efficiency parameters are the power requirements of components related to plant operation such as the vacuum system, coolant pumps, and magnet system. 
The input power $P_{i}$ into the power-source subsystem is the product of the circulating power $P_{c}$ and the weighted efficiency $\eta_{i}$ of the complex of the plasma-confinement and -heating subsystem and the injection-heating subsystem.

$$
\mathbf{P}_{\mathbf{i}}=\eta_{\mathbf{i}} \mathbf{P}_{\mathbf{C}^{*}}
$$

For the two plasma-preparation subsystems shown in Fig. 2 .

$$
T_{i}=f_{H} \eta_{H}+f_{I} \eta_{I},
$$

where $f_{H}$ is the fraction of the cixculating power necessary for the plasmaconfinement and -heating subsystem having an efficiency $\eta_{H}$, and $f_{I}$ is the fraction required to operate the injection-heating subsystem having an efficiency $T_{I}$, with $f_{H}+f_{I}=1$.

The gross electrical power output is the sum of the power converted by the conversion subsystems and is the product of the output power $P_{0}$ and the overall conversion efficiency $\eta_{c}$ of the complex of conversion subsystems,

$$
P_{B}=T_{C^{2}} P_{0}
$$

For the three power-conversion aubsystems shown in Fig. 2 ,

$$
\eta_{c}=\mathbf{f}_{T} \eta_{T}+f_{D} \eta_{D}+f_{M} \eta_{M} \text {. }
$$

where $f_{T}$ is the fraction of the total power output $P_{0}$ converted thermally with an efficiency $T_{T}, f_{D}$ is the fraction converted by direct conversion with an efficiency of $\prod_{D}$, and $f_{M}$ is the fraction converted by magnetic induction with an efficiency $\eta_{M}$, with $f_{T}+f_{D}+f_{M}=1$.

The above treatment applied to the typical syotem of Fig. 2 is equally applicable to even more complex systems, with the overall system requirementa still constrained by Eq. 4 .

\section{LIMIT TESTS}

The power-balance relations derived from the above definltions are found to be consistent with limit tests for idealized systems. One such limit is obtained by considering an idealized reactor system in which all power-conversion and -injection subsystems are designed with $100 \%$ efficiencies: i.e.. $\eta_{i}=\eta_{c}=1$. In this limiting case, the $Q$ factor from Eq. 4 becomes

$$
\lim _{\eta_{i}, \pi_{c} \rightarrow 1} Q=\frac{1}{e}
$$


From Eq. 9, the $Q$ factor in the above limit may also be written as

$$
\lim _{i, \eta_{c} \rightarrow 1} Q=1+\frac{P_{e}}{P_{c}}
$$

which shows that for a given net electrical power output, high-Q-value re* actor syotems have relatively low circulating-powes requirements.

For a break-even reactor system, that is, a zero net electrical power-producing system $\left(P_{e}=0\right)$, the critical $Q$ factor $Q_{c \text { rit }}$ is obtained by taking the limit of $Q$ as approaches unity.

$$
\lim _{\varepsilon \rightarrow 1} Q \equiv Q_{c r i t}=\frac{1}{\pi_{i} \eta_{c}}=\frac{1}{\eta}
$$

- Equations 7 and 18 show the relationship between the system efficiency $\eta$ and the power ratio $P_{f} / P_{i}$ necessary to achieve a break-even system. As an example, for an overall efficiency of $\eta_{1}=1 / 3 \mathrm{as}$ in the Lawson paper, ${ }^{2} Q_{\text {crit }}=3$, so that Lawson's parameter $R=P_{f} / P_{i}=2$; that is, the fusion power source must be twice the input power for a break-even condition.

For the combined limiting cases of $100 \%$ efficiencies and $100 \%$ fractional circulating power, the $Q$ factor from $E q .4$ becomes

$$
\lim _{\varepsilon \rightarrow 1} \lim _{\eta_{i}, \eta_{c} \rightarrow 1} Q=1
$$

This limiting $Q$ value of unity represents a theo retical self-sustaining system in which the fusion power $P_{f}$ approaches zero, and $P_{i}=P_{0}=P_{g}=P_{c}$.

\section{COMPARISON WITH OTHER Q DEFINITIONS}

Several other definitions of $Q$ have been used in a variety of spe. cific studies relating mostly to simple and minimum-B mirror machines. In a study of mirror systems incorporating direct-conversion subsystems, Post ${ }^{3}$ introduced the quantity QEN defined as

$$
Q_{E N}=\frac{\text { Converted Nuclear Power Output }}{\text { Net Required Input Electrical Power }}=\frac{P_{g}-\eta_{D} P_{j}}{P_{i} / \eta_{i}-\eta_{D} P_{i}}
$$

where all terms are defined in Sec. 2. Note that the numerator of Eq. 20 represents the total converted electrical power $\mathrm{P}_{\mathrm{g}}$ minus the directconverted input power $\prod_{D} P_{i}$ i the denominator represents the total input power into the plasma-preparation subsystem $P_{i} / \eta_{i}$ minus the directconverted input power. It is significant that the subtrahend in both the 
numerator and denominator represents the main fraction of the circulating input power required to maintain the reactor system. In effect, $Q_{E N}$ represents the $Q$ for'a subsystem of the total reactor system. As a result, QEN for the subsystem is greater than the $Q$ given by Eq. 2 for the total system.

A consequence of the definition of $Q_{E N}$ is that for the limiting case, in which the injection and conversion efficiencies approach unity, QEN becomes infinite independently of $e$. This may be seen directly from Eq. 20 , or by using the definitions of $\epsilon$ and $P_{i}$, given by Egs. 3 and 12, respectively, in Eq. 20 to give

$$
Q_{E N}=\frac{1-\varepsilon \eta_{i} \eta_{D}}{\varepsilon\left(1-\eta_{i} \eta_{D}\right)} .
$$

In this same limiting case, $Q$ as written in Eq. 4 approaches the limit of $1 / \varepsilon$ as in Eq. 16, a result which is consigtent with the basic powerflow diagram (Fig. 1) tor a total reactor power system as discussed insec. 3 . In the limit of zero net electrical power output, that is $\varepsilon \rightarrow 1$, the critical QEN value becomes unity independently of subsystem efficiencies.

The delinition of $Q_{E N}$ does not provide a measure that is indicative of the total plasma-preparation power required to maintain the total reactor system operable. As a result, there is no corresponding measure of the capital investment in equipment required to handle the total circulating power, as will be discussed in Sec. 5.

The upper section of Table 1 lists the QEN values of Ref. 3 for the three fuel cycles (1) D-T, (2) D-D, and (3) D- ${ }^{3} \mathrm{He}$, and for each the cases $A$ and $B$ corresponding to high and low collisional loss rates, respectively.

TABLE I. Values of $Q$ and E for Misror Reactors

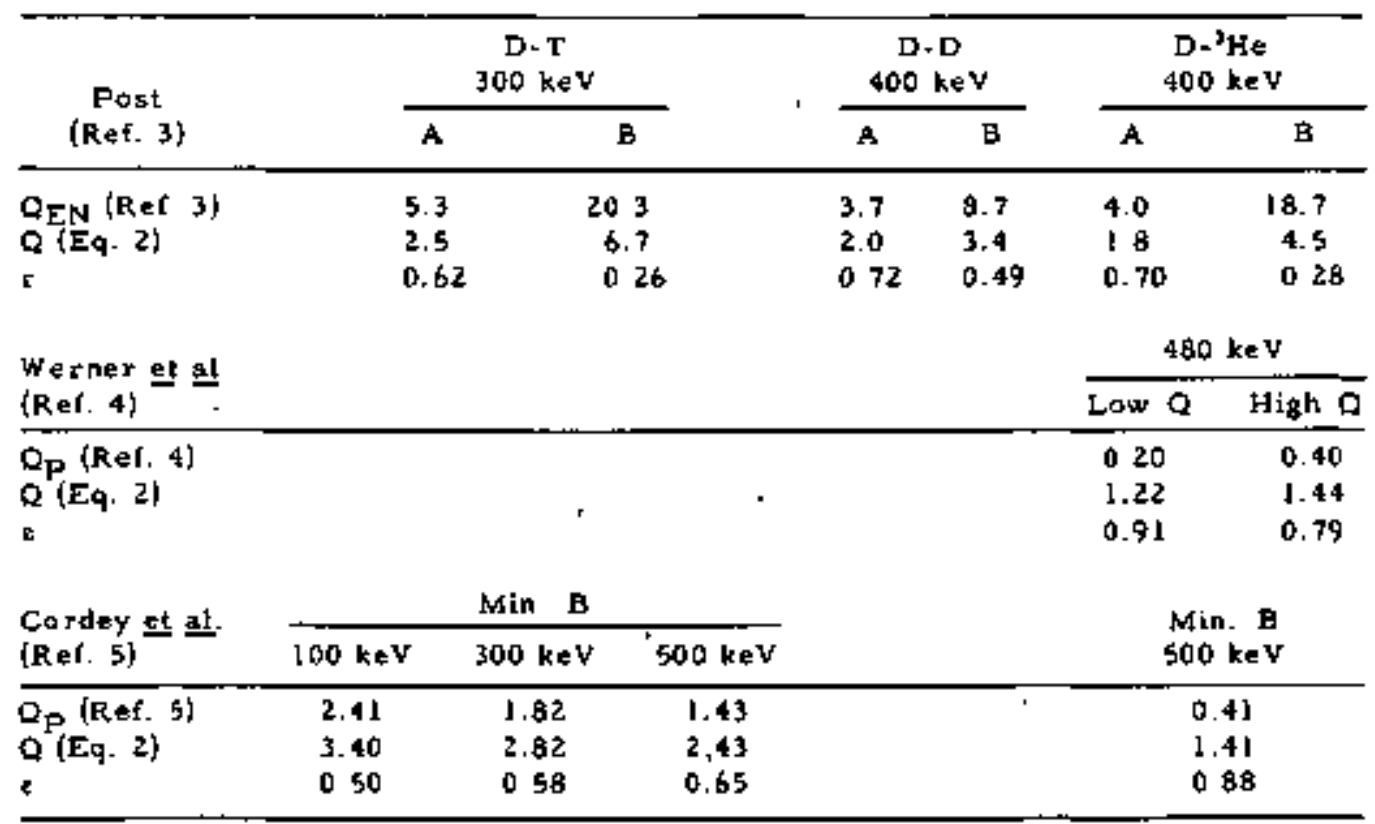


Also listed are the values of $Q$ determined from Eq. 2 using the data of Ref. 3. The corresponding values of $\varepsilon$ computed from Eq. 3 have, also been listed in Table $I$. The values of $Q_{\text {EN }}$ are found to exceed $Q$ by factors in the approximate range of 2-4.

In an earlier survey study of simple mirror machines, Post ${ }^{\dot{8}}$ introduced the quantity $Q$ defined as the ratio of total nuclear power released to the injection-particle energy escaping,*

$$
Q_{P}=\frac{\text { Reactor Nuclear Power Output }}{\text { Total Reactor Power Input }}=\frac{P_{f}}{P_{i}}
$$

where $P_{f}$ and $P_{i}$ are defined in Sec. 2. This definition involves only part of the power flow across interface $B$ of Fig. 2 , and consequently $Q$ from Eq. 7 differs from $Q_{P}$ by an additive constant of unity. In terms of $a$ and subsys tem efficiencies, Eq. 22 becomes

$$
Q_{P}=\frac{1-\epsilon \eta_{i} T_{c}}{\epsilon T_{i} \eta_{l_{c}}}
$$

The above definition of $Q$ was used in a study of minimum-B mirror machines by Fowler and Rankin ${ }^{7}$ and in a review paper by Fowler." Most reçently, this definition was used as the figure of merit in the mirror reactor power system studies by Werner et a‥ " by Cordey et al. , 5 and in a slightly modified form by Sweetman. ${ }^{9}$

The difference between the $Q_{P}$ of Eq. $2 Z$ and the $Q$ of Eq. 2 is that the injection power, $P_{i}$, is not included in the accounting for the total power output; $Q_{P}$ corresponds to Lawson's factor $R$. A consequence of the above definition is that in the limiting case of $100 \%$ injection and conversion efficiencies, $\Omega_{\mathrm{P}}$ approaches the value $(1-\epsilon) / \varepsilon$ which is equivalent to the ratio of the net electrical power output to the circulating power. Although this may appear not to be inconsistent with the corresponding limiting value $Q=1 / \mathrm{e}$ in Eq. 16, it does not represent the total power flow across interface $B$ between the power bource and the power-conversion subsystems of Fig. 1 or 2.

In the limiting case of zero net electrical power output, $\varepsilon \rightarrow 1$, the critical $Q_{\mathrm{P}}$ approaches $(1-\eta) / \eta$, rather than $1 / \pi$ as in Eq. 18 . For the combined limiting case of $\varepsilon \rightarrow 1$ and $\eta_{1} \rightarrow 1$, a self-circulating power balance (that is, reactor output power equal to reactor input power) is more appropriately described by a $Q$ value of unity, whereas the $Q_{P}$ approaches zero.

Table I lists the $Q_{P}$ values reported in the studies of Werner et al ${ }^{4}$ and Cordey et al. ${ }^{5}$ using the definition given by Eq. 22 . Values of $Q$

The notation $\mathrm{QP}$ is incroduced to distingutsh this definition from the $Q$ defined by EQ. 2 . 
determined from Eq, 2 are included for comparison. Also listed are values of calculated from Eq. 3 using data in the references cited.

\section{REMARKS}

The trend noted in Table $\mathrm{I}$ (from Ref. 5) for the D-T fuel cycle is that $Q$ and $\varepsilon$ reflect the increase in circulating-power requirements as the initial plasma-temperature conditions are increased. The Ref. 3 studies also indicate that a reduction in the end losses of a mirror machine (case $B$ versus case $A$ ) results in higher-Q-value reactor systems with corresponding reduction in circulating-powe $r$ requirements. The operating and capital costs of the reactor systems should be expected to relate to the power parameters. To a first approximation, total power costs can be assumed to scale directly with the gross electrical power output $P_{g}$. Consequentiy, the normalized capital costs are proportional to the normalized power $\mathrm{Pg}_{\mathrm{g}} / \mathrm{Pe}$ and in turn are related to $E$ through Eq. 8 as

$$
\text { Normalized Capital Costs } \alpha \frac{P_{g}}{P_{e}}=\frac{1}{1-\varepsilon} \text {. }
$$

Therefore, high capital costs are to be expected for those systems having high fractional circulating-power requirements.

The definitions of the power-balance parameters $Q$ and 6 presented in this report are applicable to complex systems involving any combination of energy conversion, plasma formation, and injection devices, and can be used as measures of performance common to all fusion-reactor systems. including closed magnetic systems or open-ended magnetic systems. Some typical values of $Q$ and $e$ listed in the survey for closed systems are as follows: stellarators, $Q \approx 13, \epsilon=0.22$; tokamaks, $7 \leq Q \leq 100,0.03 \leq$ $\varepsilon \leq 0.3$. Typical values for open-ended systems other than the mirror machine are as follows: $\theta$-pinch, $Q \approx 4.4, \varepsilon=0.36$ : astron $Q \approx 8.5$, $c=0.2$.

In summary, the power-balance parametera defined in this study serve two purposes: (1) to establish a common bas is for comparison of the fundamentally different approaches to a fusion-power-reactor system (including fusion by laser ignition); and (2) to serve eventually as feedback information to help orient and guide research and development efforts toward optimum total systems within each basic concept and for different fuel cycles. 


\section{REFERENCES}

1. P. J. Perstant, W. C. I1pinskt, and A. J. Hatch, Survey of Thermonuclear Reactor Parameters, ANL-7807 (to be published).

2. J. D. Lawson, Proc. Phys. Soc. B 70, 6 (1957).

3. R. F. Post, "Mirror Systens: Fuel Cycles, Loss Reduction and Energy Recovery," Proc. Conf. Nuctear Fusion Reactors, Culham, UKAEA, p. 88 (1969).

4. R. W. Werner, G. A. Carlson, J. D. Lee, R. W. Moir, R. P. Post, and C. E. Taylor, "Engineering and Economic Aspects of Mirror Machine Reactors with Direct Conversion," Proc. IAEA 4th Conf. PLasma Physics and Controlied Mutear Fusion Research, Madison, W1sconsin, Paper No. IAEA/ $\mathrm{CN}-28 / \mathrm{K}-2$ (1971) (to be published).

5. J. G. Cordey, F. B. Marcus, D. R. Sweetman, and C. J. H. Wation, "Efficient Recirculation of Power in Mirror Reactors," Proc. IAEA 4th Conf. Plasma Physics and Controlled Nuclear Fusion Research, Madison, Wisconsin, Paper No. IAEA/CN-28/K-3 (1971) (to be published).

6. R. F. Post, Muol, Fusion Suppl., Pt. 1, p. 99 (1962).

7. T. K. Fowler and M. Bankin, J. Nucl, Energy C 8,121 (1966).

8. T. K. Fowler, Nuct Fusion 9, 3 (1969).

9. D. R. Sweetman, "Mirror Reactors: Sone General Considerations," Proc. Conf. Wuctear Busion Reactors, Culham, UKAEA, p. 112 (1969). 